\title{
Monte Carlo with Absorbing Markov Chains: Fast Local Algorithms for Slow Dynamics
}

\author{
M. A. Novotny \\ Supercomputer Computations Research Institute, Florida State University, Tallahassee, FL \\ 32306-4052
}

(October 9, 2018)

\begin{abstract}
A class of Monte Carlo algorithms which incorporate absorbing Markov chains is presented. In a particular limit, the lowest-order of these algorithms reduces to the $n$-fold way algorithm. These algorithms are applied to study the escape from the metastable state in the two-dimensional square-lattice nearest-neighbor Ising ferromagnet in an unfavorable applied field, and the agreement with theoretical predictions is very good. It is demonstrated that the higher-order algorithms can be many orders of magnitude faster than either the traditional Monte Carlo or $n$-fold way algorithms.
\end{abstract}

02.70.Lq, 05.50.+q, 64.60.My, 75.40.Mg

Typeset using REVTEX 
Monte Carlo (MC) methods [1] have become indispensible tools for nonperturbative calculations in numerous fields, including materials science, high-energy physics, chemistry, biology, engineering, and economics. These methods are used for two fundamentally different purposes: to calculate time-independent quantities (statics) and to simulate time series (dynamics). In the former case, the slow relaxation observed, e.g. near phase transitions (critical slowing down) and at low temperatures, is merely a nuisance that has been overcome by a number of new MC algorithms, including cluster algorithms [2], vertex algorithms [3], multicanonical algorithms [4], and hybrid MC algorithms [5]. Such algorithms can be many orders of magnitude faster than standard MC methods. However, they all replace the standard MC dynamic with a different dynamic. Consequently, although such algorithms may be very efficient in the calculation of static quantities, information about the kinetics of the original MC dynamic cannot be obtained. There are many instances where the kinetics, rather than just the statics, is of physical importance. Recently, MC methods using constrained cluster-flipping algorithms have been proposed in order to obtain information about the long-wavelength kinetics of a system [6]. However, in such methods the local dynamic is modified, and universality arguments must be made to relate the results to the dynamic of the original system.

In this Letter, a different class of accelerated MC algorithms is presented that does not change the original MC dynamic, and can therefore be used to simulate time series with very large separations in time scales. These algorithms incorporate into the standard MC algorithm absorbing Markov chains (AMC). The acronym MCAMC stands for Monte Carlo with absorbing Markov chains. With some additional approximations, the lowestorder MCAMC algorithm corresponds to the $n$-fold way algorithm [7]. We demonstrate that MCAMC algorithms can be many orders of magnitude faster than either the standard MC or $n$-fold way algorithms.

In a Markov process the probability of going from the current state of the system to another state depends only on these two states, not on the history of how the current state was reached. Standard MC algorithms are typical examples of Markov processes. A Markov 
process with a finite state space and a discrete time step is a Markov chain. The Markov chain is often written as a Markov matrix, $\mathbf{M}$, the elements of which are the transition probabilities between the states. The time evolution of the probability distribution vector $\vec{v}^{\mathrm{T}}$ is then given by $\vec{v}^{\mathrm{T}}(m+1)=\vec{v}^{\mathrm{T}}(m) \mathbf{M}$. An AMC is one in which at least one state has the property that transitions out of that state are forbidden.

The Markov matrix associated with an AMC with $q$ absorbing states and $s$ transient states is given by 8

$$
\mathbf{M}=\left(\begin{array}{cc}
\mathbf{I}_{q \times q} & \mathbf{0}_{q \times s} \\
\mathbf{R}_{s \times q} & \mathbf{T}_{s \times s}
\end{array}\right),
$$

where $\mathbf{I}$ is the identity matrix, $\mathbf{0}$ is the zero matrix, and the subscripts show the size of each submatrix. The elements of the transient submatrix, $\mathbf{T}$, are the transition probabilities between the $s$ transient states of $\mathbf{M}$. The elements of the submatrix $\mathbf{R}$ are the transition probabilities from each of the $s$ transient states to the $q$ absorbing states. Starting from an initial vector $\vec{v}_{I}^{\mathrm{T}}$, the probability of still being in any of the $s$ transient states after $m$ time steps is given by $\vec{v}_{I}^{\mathrm{T}} \mathbf{T}^{m} \vec{e}$, where $\vec{e}$ is the vector with all elements equal to 1 . Although $\vec{v}_{I}^{\mathrm{T}}$ could be any normalized probability distribution over the transient states, in this work we will take $\vec{v}_{I}^{\mathrm{T}}$ to represent one particular transient state. It is easy to show that one can obtain the time $m$ for exiting to one of the $q$ absorbing states from the solution of the equation

$$
\vec{v}_{I}^{\mathrm{T}} \mathbf{T}^{m} \vec{e}<r \leq \vec{v}_{I}^{\mathrm{T}} \mathbf{T}^{m-1} \vec{e}
$$

where $r$ is a random number uniformly distributed on the interval $(0,1]$. Note that the stochastic variable $m$ is independent of which of the $q$ states the AMC ends up in. The elements of the vector of $q$ unnormalized probabilities

$$
\overrightarrow{\mathcal{Q}}^{\mathrm{T}}=\vec{v}_{I}^{\mathrm{T}} \mathbf{T}_{s \times s}^{m-1} \mathbf{R}_{s \times q}
$$

give the probability of exiting to each corresponding state, given that the system has exited from the transient subspace in $m$ time steps. After normalization, this probability distribution can be used to pick a particular state into which the AMC exits. This is done by 
using another uniformly-distributed random number. Eqs. (2) and (3) are the only equations needed to include absorbing Markov chains within a Monte Carlo simulation. In practice, it is computationally sound policy to obtain all the eigenvalues and eigenvectors of $\mathbf{T}$ and utilize the spectral decomposition of $\mathbf{T}$ within the AMC portion of the algorithm to numerically solve Eqs. (2) and (3).

The MCAMC algorithm has the following steps: 0) The system is in an initial state represented by $\vec{v}_{I}^{T}$. 1) Write an $s \times s$ transient matrix that includes at least the initial configuration, but ideally will include other states (which may represent many configurations of the system that are related by symmetry). The additional states included as transient states should be those the current configuration has the largest probability of exiting to. 2) All configurations that the transient states can exit to in one time step must be included in the absorbing states. 3) Use Eq. (2) to find the time spent in the transient state subspace, and Eq. (3) to find a new configuration for the system. 4). Iterate the procedure using the new configuration as the next initial configuration. Thus, once the system has exited to one of the $q$ absorbing states, this decides the initial configuration of the system for a different AMC with different transient and absorbing subspaces, and the process is iterated.

To illustrate the MCAMC algorithm, we apply it to the square-lattice nearest-neighbor Ising ferromagnet in a magnetic field. The Hamiltonian is given by $\mathcal{H}=-J \sum_{\langle i, j\rangle} s_{i} s_{j}-$ $H \sum_{i} s_{i}$, where the spins $s_{i}= \pm 1$. The sums run over all nearest-neighbor pairs and over all $N=L^{2}$ sites, respectively. Periodic boundary conditions are used. The isotropic two-body coupling constant is given by $J>0$ and the magnetic field by $H$. To study the decay of a metastable phase, we apply a negative magnetic field at a temperature below the critical temperature $T_{c}$, and start with the configuration of all spins +1 (which we call the $\mathrm{C}+$ configuration). Standard droplet theory (for a recent review on metastability see Ref. [9]) shows that in order for the metastable phase $\mathrm{C}+$ to decay, one or more critical droplets must nucleate via the random superposition of microscopic fluctuations. Since the critical droplet is of a certain size, the average time before its creation, and thus the metastable lifetime, is much longer than the microscopic timescales. Although any local dynamic can be used 
within the MCAMC framework, in this letter only Metropolis updates [1] are performed. We measure the number of Monte Carlo steps per spin (MCSS) until a configuration is reached which has an equal number of +1 and -1 spins. The average lifetime, $\tau$, of the metastable state is found by averaging over a number of starts from the $\mathrm{C}+$ configuration.

A standard MC algorithm randomly chooses a spin, and then decides whether or not to flip it. Each spin in the $L \times L$ lattice, with periodic boundary conditions, is in one of 10 possible energy classes which are determined by how the spin is oriented with respect to the applied field and the nearest-neighbor spins [7]. The number of spins in class $i$ is $n_{i}$, and the probability of flipping a spin in class $i$ once it has been chosen is $p(i)$.

The $s=1 \mathrm{MCAMC}$ algorithm is defined to have a single transient state, which is the current state of the spin configuration. When spins belonging to each class are grouped together the submatrix $\mathbf{R}_{1 \times 10}=\left(n_{1} p(1), \cdots, n_{10} p(10)\right) / N$. Define $Q_{0}=0$ and $Q_{j}=\sum_{i=1}^{j} n_{i} p(i)$. Since $\mathbf{M}$ is a Markov matrix, its row-sums are unity, so the transient matrix is $\mathbf{T}_{1 \times 1}=1-Q_{10} / N$. The time increment to flip a spin is found from Eq. (2) to be $m>\ln (r) / \ln \left(1-Q_{10} / N\right) \geq m-1$. If one relaxes the constraint that $m$ is an integer, and expands to lowest order for small $Q_{10} / N$, this equation becomes $\widehat{m}=-N \ln (r) / Q_{10}$, and one has the standard $n$-fold way algorithm [7]. In both the $s=1 \mathrm{MCAMC}$ and the $n$-fold way algorithm, another random number is used to choose which of the 10 classes to pick via $Q_{j-1}<r Q_{10} \leq Q_{j}$, and a spin from class $j$ is randomly picked and flipped. As shown in Fig. 目, at low temperatures the $s=1$ MCAMC algorithm can be many orders of magnitude faster than the standard MC algorithm. At $J / T=3$ the speed improves by a factor of about $10^{7}$.

At low temperatures in the $s=1 \mathrm{MCAMC}$ algorithm, once a spin has flipped from the $\mathrm{C}+$ configuration, it is extremely probable that in the next iteration this spin will be chosen again to flip, and one returns to the $\mathrm{C}+$ configuration. This problem can be addressed by going to the $s=2 \mathrm{MCAMC}$ algorithm. Whenever the starting configuration is the $\mathrm{C}+$ configuration or a configuration with only one spin overturned from the $\mathrm{C}+$ configuration, one includes these two states in the transient matrix $\mathbf{T}$. The normal $s=1$ MCAMC algorithm is used if the spin configuration is other than one of the transient states. As shown in Fig. 
1, for $J / T=3$ the speed improves by a factor of about $10^{2}$ over the $n$-fold way algorithm.

One can continue to increase the number of states in T. Figure 1 also includes results from $s=3$ MCAMC, where the three states in $\mathbf{T}$ were $\mathrm{C}+, \mathrm{C}+$ with one overturned spin, and $\mathrm{C}+$ with two nearest-neighbor overturned spins. At $J / T=3$ this $s=3 \mathrm{MCAMC}$ algorithm improves the speed by a factor of about $10^{2}$ compared to the $s=2$ MCAMC algorithm.

The MCAMC algorithm offers a further advantage, in that the statistical error in the average lifetime $\tau$ is smaller than from standard MC. The average lifetime of an AMC that starts from state $k$ is given by $\vec{v}_{k}^{\mathrm{T}} \mathbf{N} \vec{e}$, where the fundamental matrix is defined by $\mathbf{N}=(\mathbf{I}-\mathbf{T})^{-1}[8]$. For $s>1$, the initial configuration in which the AMC starts is C+ only once, since it must re-enter $\mathrm{C}+$ from a configuration $\mathrm{C}+$ with one overturned spin. Since the contribution to the lifetime that comes from the escape from $\mathrm{C}+$ is exact, this reduces the total error in $\tau$.

It has been shown that at low enough temperatures $\tau$ is related to the height of the lowest energy barrier which must be reached by flipping one spin at a time starting from $\mathrm{C}+[10]$. In the limit of low temperature and large $L$, the discreteness of the lattice gives an important contribution to $\tau$. Theorem 3 of Ref. [10] states that then

$$
k_{B} T \ln \left(L^{2} \tau\right)=\Gamma(H, J)=8 J \ell_{c}-2|H|\left(\ell_{c}^{2}-\ell_{c}+1\right)
$$

where $\ell_{c}=\lceil 2 J /|H|\rceil$ and the notation $\lceil x\rceil$ denotes the smallest integer larger than $x$. This result, which is restricted to $2 J /|H|$ not an integer and to $|H|<4 J$, is shown in Fig. 1 as a solid line. It is in qualitative agreement with the measured values of $\tau$. A detailed comparison with predictions from Ref. [10] will be published elsewhere [11].

Using similar reasoning, it is possible to estimate at low temperatures the temperature dependence of the different MCAMC algorithms. By assuming that most of the CPU time is spent in the $s=1$ portion of the algorithm, at low enough temperatures the CPU time should be proportional to $\exp \left\{\left[\Gamma(H, J)-\Gamma_{0}(H, J)\right] / k_{B} T\right\}$. Here $\Gamma_{0}$ is $2 J$ times the surface area minus $2|H|$ times the volume of the largest compact lattice animal included in $\mathbf{T}$. The dashed lines in Fig. 11 have these slopes and are drawn to go through the data point at the 
lowest temperature available for a particular algorithm.

Figure 2 shows values for $\tau$ as a function of $|H|$ for two low temperatures. Note how well Eq. (田) fits the results. Eq. (丰) is only valid if the nucleation of a single droplet is responsible for flipping the lattice into the stable phase. A reasonable estimate for the crossover field (which has been called the dynamic spinodal field [12]) out of the single-droplet regime is $H_{1 / 2}$, the field at which the standard deviation of the lifetime is $\tau / 2$ [12]. Fig. 2 shows that this is a reasonable estimate for the point at which the results deviate from Eq. (4) at strong fields.

At higher temperatures, the discreteness of the lattice becomes less important, and $\tau$ should be given by the continuum droplet-theory prediction [13] 16$]$

$$
\ln (\tau)=\Xi(T) /|H|-(b+c) \ln |H|+\ln (A(T))
$$

Here $\Xi(T)$ is related to the equilibrium zero-field surface tension, the equilibrium droplet shape [17], and the spontaneous magnetization. $A(T)$ is a non-universal function. Fieldtheoretical and numerical calculations give $b=1$ [14,16, 18]. For dynamics described by a Fokker-Planck equation $c=2$ [15, [16], and a recent MC study is consistent with $b+c=3$ [12].

Differentiating Eqs. (4) and (5) with respect to $|H|^{-1}$ allows for direct comparisons with both the discrete droplet and continuum droplet predictions. This is shown in Fig. 3. For $T=0.4 J$ a crossover is observed at weak $|H|$ to the continuum droplet prediction using $b+c=3$ and the exact zero-field value for $\Xi(T)$ [17]. At weak $|H|$ Fig. 3 shows that the $T=0.4 J$ data are consistent with the theoretical predictions of Eq. (5) with the theoretical values for $\Xi(T)$ and $b+c$.

MCAMC algorithms can be utilized to study other interesting low-temperature effects in lattice-gas types of models. An example is the recent prediction that at low enough temperatures in the anisotropic Ising model nucleation occurs through square droplets, rather than through rectangular equilibrium Wulff droplets [19].

Additional applications of MCAMC algorithms may include studies of equilibrium properties of systems where the $n$-fold way algorithm has been utilized. These include the 
anisotropic Ising model [20] (which is related via a Trotter-Suzuki decomposition to a quantum model in one lower dimension [21]), and simulated annealing approaches to minimization [22].

However, the real strength of MCAMC algorithms lies in the study of slow dynamics in models with a limited number of local states. MCAMC algorithms will be particularly useful when there exists a small number of spins which are rapidly fluctuating, and major rearrangements of spins occur very infrequently. Examples would be the kinetics of spinglass models [25], where the spins that fluctuate rapidly are the ones that find themselves in a local environment with zero energy difference between the possible spin orientations. Another example is phase-ordering kinetics in which a few particles undergo rapid random walks on long flat portions of interfaces, while the interfaces move extremely slowly. Similar reasoning also applies to simulations of molecular-beam epitaxial growth, where the $n$-fold way algorithm has been rediscovered at least twice 23,24. In all such cases, using higher-

order MCAMC algorithms should substantially decrease the CPU time required to obtain the static and dynamic information about the system without making any approximations to the dynamics.

\section{ACKNOWLEDGMENTS}

The author wishes to thank J. Lee and P. A. Rikvold for useful discussions and suggestions. This research was supported in part by the Florida State University Supercomputer Computations Research Institute, which is partially funded through contract \# DE-FC0585ER25000 by the U.S. Department of Energy. 


\section{REFERENCES}

[1] K. Binder and D. Stauffer, in Applications of the Monte Carlo Method in Statistical Physics, in Topics in Current Physics, Vol. 36, edited by K. Binder (Springer-Verlag, Berlin, 1984).

[2] For a review see: A. D. Sokal in Lattice '90, [Nucl. Phys. B (Proc. Suppl.)] 20, 216 (1991).

[3] H. G. Evertz, G. Lana, and M. Marcu, Phys. Rev. Lett. 70, 875 (1993).

[4] B. A. Berg and T. Celik, Phys. Rev. Lett. 69, 2292 (1992).

[5] S. Duane, A. D. Kennedy, B. J. Pendleton, and D. Roweth, Phys. Lett. B 195, 216 (1986).

[6] G. T. Barkema and J. F. Marko, Phys. Rev. Lett. 71, 2070 (1993).

[7] A. B. Bortz, M. H. Kalos, and J. L. Lebowitz, J. Comput. Phys. 17, 10 (1975).

[8] M. Iosifescu, Finite Markov Processes and Their Application, Chapter 3, (John Wiley \& Sons, New York, 1980).

[9] P. A. Rikvold and B. M. Gorman, Annual Reviews of Computational Physics, Vol. 1, edited by D. Stauffer, (World Scientific, Singapore, 1994).

[10] E. Jordão Neves and R. H. Schonmann, Commun. Math. Phys. 137, 209 (1991).

[11] M. A. Novotny, unpublished.

[12] P. A. Rikvold, H. Tomita, S. Miyashita, and S. W. Sides, Phys. Rev. E, 49, 5080 (1994).

[13] M. E. Fisher, Physics 3, 255 (1967).

[14] J. S. Langer, Ann. Phys. (N.Y.) 41, 108 (1967).

[15] J. S. Langer, Ann. Phys. (N.Y.) 54, 258 (1969). 
[16] N. J. Günther, D. A. Nicole, and D. J. Wallace, J. Phys. A 13, 1755 (1980).

[17] R. K. P. Zia and J. E. Avron, Phys. Rev. B 46, 8886 (1992).

[18] C. C. A. Günther, P. A. Rikvold, and M. A. Novotny, Phys. Rev. Lett. 71, 3898 (1993).

[19] R. Kotecky and E. Olivieri, J. Stat. Phys. 70, 1121 (1993).

[20] T. Graim and D. P. Landau, Phys. Rev. B 24, 5156 (1981).

[21] M. Suzuki in Quantum Monte Carlo Methods in Condensed Matter Physics, edited by M. Suzuki (World Scientific, Singapore, 1993).

[22] J. W. Greene and K. J. Supowit, IEEE Trans. on Computer-Aided Design CAD-5, 221 (1986).

[23] G. H. Gilmer, J. Crystal Growth 35, 15 (1976).

[24] P. A. Maksym, Semicond. Science Technol. 3, 594 (1988).

[25] After completion of this work, two similar recent works were found: W. Krauth and M. Mézard, to appear in Z. Phys. B (LPTENS preprint 94/20); W. Krauth and O. Pluchery, LPTENS preprint (1994). 


\section{FIGURES}

FIG. 1. The average CPU time for escape from the metastable state is shown as a function of the inverse temperature. This is for a $24 \times 24$ lattice in a field of $|H| / J=0.75$. For the standard Monte Carlo algorithm $(\times)$ the CPU time is directly proportional to the average lifetime $\tau$ of the metastable state, and $\tau$ (in units of Monte Carlo steps per spin) is plotted on the right-hand axis. All values of $\tau$ were calculated by averaging over $10^{3}$ escapes from the metastable state. The symbol $\times$, with error estimates, for $J / T \geq 1$ is calculated from the values of $\tau$ from the MCAMC algorithms, while for $J / T<1$ the CPU time for a standard Monte Carlo algorithm is plotted. The CPU time from the $n$-fold way algorithm, which corresponds to $s=1$ MCAMC, is given by the symbol $\diamond$. The timings for $s=2$ and $s=3$ MCAMC algorithms are given by the symbols + and $\square$, respectively. The vertical arrow marks the exact critical temperature. The solid line is the low-temperature discrete droplet result [10] for $\tau$ with $\ell_{c}=3$ from Eq. (4). The dashed lines are estimates, described in the text, for the CPU times for the MCAMC algorithms. The horizontal arrows indicate that the solid line and symbols $\times$ are related to both vertical axes, whereas the other points and lines are only for the left-hand axis. The curvature near $J / T \approx 1$ is near the value where $H_{1 / 2}=3 J / 4$ for this system size. Note the spectacular increases in speed with the MCAMC algorithms.

FIG. 2. The average lifetime $\tau$, as a function of $|H|$, for escape from the metastable state is shown for a $24 \times 24$ lattice with $T / J=0.2\left(T / T_{c} \approx 0.088\right)(\times)$ and $T / J=0.4(\bigcirc)$. Error estimates are plotted for each point. The solid lines are the low-temperature discrete droplet estimates for $\tau$ from Eq. (四). The diagrams show the nucleating droplet, reading from the strongest fields with $\ell_{c}$ of 1,2 , and 3 . The dashed lines show the boundary between the various $\ell_{c}$ regions. The vertical arrows mark the cross-over field $H_{1 / 2}$ [12], which separates the single-droplet regime $\left(|H|<H_{1 / 2}\right)$ and the multidroplet regime $\left(H_{1 / 2}<|H|\right)$ for the two temperatures for this lattice size. 
FIG. 3. The temperature times the derivative of the logarithm of the average lifetime with respect to $|H|^{-1}$ is shown as a function of $|H|$. This is for a $24 \times 24$ lattice with $T=0.2 J(\times)$ and $T=0.4 J(\bigcirc)$. The solid curves are from the low-temperature predictions 10 from Eq. (荬. Only results in the single droplet regime, $|H| \leq H_{1 / 2}$, are shown. The dot-dashed and dashed horizontal lines correspond to the exact [17] zero-field value for $\Xi(T) / T$ for $T=0.2 J$ and $T=0.4 J$, respectively. The dashed inclined straight line includes the theoretical value $b+c=3$ in Eq. (5) for $T=0.4 J$. 